\title{
Mean Curvature Type Flow with Perpendicular Neumann Boundary Condition inside a Convex Cone
}

\author{
Fangcheng Guo, Guanghan Li, and Chuanxi Wu \\ School of Mathematics and Statistics, Hubei University, Wuhan 430062, China \\ Correspondence should be addressed to Guanghan Li; liguanghan@163.com \\ Received 9 January 2014; Accepted 30 June 2014; Published 17 July 2014 \\ Academic Editor: Narcisa C. Apreutesei
}

Copyright ( 2014 Fangcheng Guo et al. This is an open access article distributed under the Creative Commons Attribution License, which permits unrestricted use, distribution, and reproduction in any medium, provided the original work is properly cited.

We investigate the evolution of hypersurfaces with perpendicular Neumann boundary condition under mean curvature type flow, where the boundary manifold is a convex cone. We find that the volume enclosed by the cone and the evolving hypersurface is invariant. By maximal principle, we prove that the solutions of this flow exist for all time and converge to some part of a sphere exponentially as $t$ tends to infinity.

\section{Introduction}

Let $\mathbb{N}^{n+1}(K)$ be a space form of sectional curvature $K=1,0$, or -1 . It is well known that the Riemannian metric of $\mathbb{N}^{n+1}(K)$ can be defined as

$$
d s^{2}=d \rho^{2}+\phi^{2}(\rho) d z^{2}
$$

where $d z^{2}$ is the standard induced metric of unit sphere $\mathbb{S}^{n}$ in Euclidean space $\mathbb{R}^{n+1}$, and

$$
\phi(\rho)= \begin{cases}\sin \rho & K=1 \\ \rho & K=0 \\ \sinh \rho & K=-1 .\end{cases}
$$

Recently, Guan and Li [1] introduced a new type flow in the above space form which was called mean curvature type flow, as follows:

$$
\frac{\partial F}{\partial t}=\left(n \phi^{\prime}(\rho)-H u\right) v
$$

where $H$ and $v$ are the mean curvature and the outward unit normal vector of the evolving hypersurfaces, respectively, and $u$ is the support function of the evolving hypersurfaces defined by $u=\langle\phi(\rho)(\partial / \partial \rho), \nu\rangle$. They proved that this flow evolves closed star-shaped hypersurfaces in space form into some sphere. A natural feature of this flow is that, along the mean curvature type flow, the volume enclosed by the evolving hypersurface is a constant and its area is always decreasing as long as the solution exists.

Inspired by this result, we focus on the corresponding problem with perpendicular Neumann boundary condition inside a convex cone in $\mathbb{R}^{n+1}$. Precisely, we suppose that $\Sigma \subset$ $\mathbb{R}^{n+1}$ is a convex cone with outward unit normal vector $\mu$ and $M^{n}$ is a smooth $n$-dimensional hypersurface with boundary $\partial M$ defined by an initial embedding $F_{0}: M^{n} \rightarrow \mathbb{R}^{n+1}$. We will study how $M^{n}$ evolves under the flow (3) with boundary conditions: $F_{0}(\partial M) \subset \Sigma$, and $\langle\mu, \nu\rangle=0$, where $\nu$ is the outward unit normal vector to $M^{n}$. Namely, we will consider the following mean curvature type flow with perpendicular Neumann boundary condition:

$$
\begin{aligned}
\frac{d F}{d t} & =(n-H u) \nu \quad \forall(y, t) \in M^{n} \times[0, T) \\
F(\cdot, 0) & =M_{0} \\
F(y, t) & \subset \Sigma \quad \forall(y, t) \in \partial M^{n} \times[0, T) \\
\langle\mu, \nu\rangle(y, t) & =0 \quad \forall(y, t) \in \partial M^{n} \times[0, T),
\end{aligned}
$$

where $u$ is the Euclidean support function to the hypersurface $M_{t}$ in $\mathbb{R}^{n+1}$ defined by $u=\langle F, v\rangle$.

We take the origin on $\mathbb{R}^{n+1}$ at the vertex of the cone, and $|F(y)|$ denotes the norm of the position vector at some point $y \in M^{n}$. In this paper, we obtain the following main result. 
Theorem 1. Suppose $\Sigma$ is a convex cone and $M_{0}$ is the initial hypersurface which can be represented as a graph over the intersection of the interior of the cone $\sum$ and a unit sphere $\mathbb{S}^{n}$ centered at the vertex of the cone; then, a solution to (4) exists for all time and stays always between two spheres with radii $R_{1}=$ $\max _{y \in M_{0}}\left|F_{0}(y)\right|$ and $R_{2}=\min _{y \in M_{0}}\left|F_{0}(y)\right|$. Furthermore, the solution converges exponentially fast to the intersection of the interior of the cone and the sphere with radius

$$
R=(n+1) \frac{|V|}{\left|M_{\infty}\right|},
$$

where $\left|M_{\infty}\right|$ denotes the area of the intersection of the limit sphere and the interior of the cone and $|V|$ denotes the volume of the domain enclosed by the cone and the evolving hypersurface which is a constant under this flow.

The MCF with boundary conditions has been extensively studied by many mathematicians. Huisken in [2] considered the evolution of a graph over a bounded domain $\Omega \subset$ $\mathbb{R}^{n}$ with perpendicular Neumann boundary condition and proved that the solution exists for all time and converges to a plane domain at last. More generally, for the hypersurfaces not necessarily represented as graphs, Stahl [3,4] studied this problem and proved that the flow converges to a round point on the condition that the boundary manifold was umbilic and the initial surface was convex. Buckland in [5] founded boundary monotonicity formulae and classified Type I boundary singularities for $H>0$ with a perpendicular Neumann boundary condition. Recently, Lambert in [6] considered this problem in a Minkowski space with a timelike cone boundary condition and proved that this flow converges to a homothetically expanding hyperbolic solution. Subsequently, he [7] also considered this problem inside a rotational tori.

However, little is known about the modified MCF, such as volume or area preserving MCF, with perpendicular Neumann boundary condition. Let $M^{n}$ be a tubular hypersurface between two parallel planes in $\mathbb{R}^{n+1}$, which can be represented as a graph over some cylinder inside it and meets the parallel planes perpendicularly. Recently, Hartley [8] studied the motion of $M^{n}$ under the volume preserving mean curvature flow by the center manifold analysis and proved that the solution exists for all time and converges exponentially fast to a cylinder in the $C^{k}$ topology for any $k \in \mathbb{N}$ as time $t$ tends to infinity.

Generally, for the prescribed contact angle which is not necessarily a right angle, this problem seems more hard. Altschuler and $\mathrm{Wu}$ in [9] considered the evolution of 2dimensional graph over compact convex domain in $\mathbb{R}^{2}$ by mean curvature flow under this boundary condition and proved this flow exists for all time and converges to translating solutions at last. Guan [10] extended AltschulerWu's result to graphs of high dimensions.

The rest of this paper is organized as follows. In Section 2, we reparametrize the system (4) as a graph and give some primary facts. In Section 3, the evolution equations and boundary derivatives for some useful geometric quantities will be derived. In Section 4, a maximal principle will be introduced and some basic estimates will be given. In the last section, we prove the convergence and complete the proof of the main theorem.

\section{Reparametrization and Notations}

Let $M^{n}$ be a compact hypersurface inside an $n$-dimensional convex cone $\Sigma$ with boundary condition $\partial M \subset \Sigma$, given by the embedding $F: \Omega \in \mathbb{S}^{n} \rightarrow \mathbb{R}^{n+1}$, where $\Omega$ is the intersection of the interior of the cone and unit sphere $\mathbb{S}^{n}$ centered at the vertex $o$ of the cone; that is, $M^{n}$ can be expressed as a graph over $\Omega$. Precisely, for any point $x \in \Omega \subset \mathbb{S}^{n}$, there is only one ray from the vertex $o$ through $x$ intersecting the hypersurface $M^{n}$ at some point $F(x)$; the position vector to $M^{n}$ can be expressed as

$$
F(x)=\rho(x) x, \quad \rho(x) \in \mathbb{R}^{+}, \quad x \in \Omega \subset \mathbb{S}^{n} .
$$

Let $\left\{x_{1}, \ldots, x_{n}\right\}$ be the local normal coordinates on $\mathbb{S}^{n} ; e_{i j}$ denotes the standard spherical metric under the coordinates; the covariant derivative and divergent operator on $\mathbb{S}^{n}$ with respect to the metric $e_{i j}$ are denoted by $\nabla$ and div, respectively. Then, tangent vectors and the outward unit normal vector on $M^{n}$ can be expressed as in [11] (see also P28 in [12]):

$$
\begin{gathered}
\nabla_{i} F=\nabla_{i} \rho x+\rho \nabla_{i} x, \\
\nu=\frac{1}{\sqrt{\rho^{2}+|\nabla \rho|^{2}}}\left(\rho x-e^{i j} \nabla_{i} \rho \nabla_{j} x\right),
\end{gathered}
$$

where $e^{i j}$ is the inverse of $e_{i j}$. Thus, the support function, induced metric, and second fundamental form can be given by straightforward calculation as following:

$$
\begin{aligned}
u & =\frac{\rho^{2}}{\sqrt{\rho^{2}+|\nabla \rho|^{2}}}, \\
g_{i j} & =\rho^{2} e_{i j}+\nabla_{i} \rho \nabla_{j} \rho, \quad g^{i j}=\frac{1}{\rho^{2}}\left(e^{i j}-\frac{\nabla^{i} \rho \nabla^{j} \rho}{\rho^{2}+|\nabla \rho|^{2}}\right), \\
h_{i j} & =\frac{1}{\sqrt{\rho^{2}+|\nabla \rho|^{2}}}\left(-\rho \nabla_{i} \nabla_{j} \rho+2 \nabla_{i} \rho \nabla_{j} \rho+\rho^{2} e_{i j}\right) .
\end{aligned}
$$

Furthermore, the system (4) is equivalent to the following parabolic PDE defined on $\Omega \times[0, T)$ :

$$
\begin{aligned}
\frac{d \rho}{d t} & =(n-H u) \frac{\rho}{u}=\operatorname{div}\left(\frac{1}{\sigma} \nabla \rho\right)+\frac{n}{\rho \sigma}|\nabla \rho|^{2} \\
\forall(x, t) \in \Omega \times[0, T), & \\
\nabla \rho \cdot \mu & =0 \quad \forall(x, t) \in \partial \Omega \times[0, T), \\
\rho(x, 0) & =\rho_{0}(x),
\end{aligned}
$$

where $\sigma=\sqrt{\rho^{2}+|\nabla \rho|^{2}}$.

The system (9) is a quasi-linear parabolic equation in divergence form, whose long-time existence is equivalent to 
the uniform parabolicity, and $C^{1}$ bound on $\rho$ by the classical theory of nonlinear parabolic equations (see, e.g., Chapter 12 in [13]).

For simplicity, let $r=\ln \rho$; then,

$$
\nabla_{i} r \rho=\nabla_{i} \rho, \quad|\nabla r|^{2} \rho^{2}=|\nabla \rho|^{2}, \quad u=\frac{\rho}{\omega},
$$

where $\omega=\sqrt{1+|\nabla r|^{2}}$. Then, the geometric quantities in (8) can be represented as

$$
\begin{aligned}
v & =\frac{1}{\omega}\left(x-e^{i j} \nabla_{i} r \nabla_{j} x\right), \\
g_{i j} & =\rho^{2}\left(e_{i j}+\nabla_{i} r \nabla_{j} r\right), \quad g^{i j}=\frac{1}{\rho^{2}}\left(e^{i j}-\frac{\nabla^{i} r \nabla^{j} r}{\omega^{2}}\right), \\
h_{i j} & =\frac{\rho}{\omega}\left(-\nabla_{i} \nabla_{j} r+\nabla_{i} r \nabla_{j} r+e_{i j}\right),
\end{aligned}
$$

and (9) can be rewritten as

$$
\begin{aligned}
\frac{d r}{d t} & =\frac{n}{u}-H=\operatorname{div}\left(\frac{1}{\rho \omega} \nabla r\right)+(n+1) \frac{|\nabla r|^{2}}{\rho \omega} \\
\forall(x, t) \in \Omega \times[0, T), & \\
\nabla r \cdot \mu & =0 \quad \forall(x, t) \in \partial \Omega \times[0, T), \\
r(x, 0) & =r_{0}(x) .
\end{aligned}
$$

System (12) is also a quasi-linear parabolic equation in divergence form, and the related estimates will be derived in Section 4.

For convenient calculation, we also parametrize the boundary cone as Lambert [6]. Let $\widetilde{S}: \mathbb{S}^{n-1} \rightarrow \mathbb{B}^{n}(0) \subset \mathbb{R}^{n}$ be a smooth embedding of a sphere into a topological ball centered at the origin with outward unit normal vector $\mathbf{n}$. Then, we can define the boundary cone $\Sigma$ by embedding $\mathbb{R}^{n}$ into $\mathbb{R}^{n+1}$ at height 1 , defining $\Sigma$ to be the set of all rays going through the origin and some point $(\widetilde{S}(z), 1) \in \mathbb{R}^{n+1}$, where $\left\{z_{1}, \ldots, z_{n-1}\right\}$ denotes the $(n-1)$-dimensional coordinate for $\widetilde{S}$. So we can parametrize the cone by

$$
F_{\Sigma}(l, \widetilde{S}(z))=\frac{l}{\sqrt{1+|\widetilde{S}|^{2}}}\left(\widetilde{S}(z)+e_{n+1}\right),
$$

where $e_{n+1}$ is the $(n+1)$ th standard coordinate vector in $\mathbb{R}^{n+1}$. The second fundamental form of the boundary cone has the following characterization.

Proposition 2. For the second fundamental form of the boundary cone, one has

(i) $A^{\Sigma}(\partial / \partial l, \cdot)=0$

(ii) $A^{\Sigma}\left(\partial \widetilde{S} / \partial z_{i}, \partial \widetilde{S} / \partial z_{j}\right)=\left(l /\left(1+|\widetilde{S}|^{2}\right)\right) A^{\widetilde{S}}\left(\partial \widetilde{S} / \partial z_{i}, \partial \widetilde{S} / \partial z_{j}\right)$, $i=1, \ldots, n-1$,

where $A^{\Sigma}$ and $A^{\widetilde{S}}$ denote the second fundamental forms, respectively, of $\Sigma$ in $\mathbb{R}^{n+1}$ and $\widetilde{S}$ in $\mathbb{R}^{n}$.
Proof. From the parametrization for the cone, it is easy to check that

$$
\left\langle\frac{\partial \widetilde{S}}{\partial z_{i}}, \mu\right\rangle=0, \quad\left\langle\widetilde{S}+e_{n+1}, \mu\right\rangle=0 .
$$

Since $\mu$ is the outward unit normal vector to the boundary cone, calculating by Gauss equation directly we have

$$
\begin{aligned}
A^{\Sigma}\left(\frac{\partial}{\partial l}, \frac{\partial}{\partial l}\right) & =h_{l l}^{\Sigma}=-\left\langle\frac{\partial^{2} F_{\Sigma}}{\partial l^{2}}, \mu\right\rangle=\langle 0, \mu\rangle=0, \\
A^{\Sigma}\left(\frac{\partial}{\partial l}, \frac{\partial \widetilde{S}}{\partial z_{i}}\right) & =h_{l i}^{\Sigma} \\
= & \left\langle\frac{1}{\sqrt{1+|\widetilde{S}|^{2}}} \frac{\partial \widetilde{S}}{\partial z_{i}}-\left(\frac{1}{\sqrt{1+|\widetilde{S}|^{2}}}\right)_{i}\right. \\
& \left.\times\left(\widetilde{S}(z)+e_{n+1}\right), \mu\right\rangle=0 .
\end{aligned}
$$

This proves the first identity.

Similarly, using (14) again we have

$$
\begin{aligned}
A^{\Sigma}\left(\frac{\partial \widetilde{S}}{\partial z_{i}}, \frac{\partial \widetilde{S}}{\partial z_{j}}\right) & =-\left\langle\frac{\partial^{2} F_{\Sigma}}{\partial z_{i} \partial z_{j}}, \mu\right\rangle \\
& =-\left\langle\frac{\partial^{2} \widetilde{S}}{\partial z_{i} \partial z_{j}}, \mu\right\rangle \frac{l}{\sqrt{1+|\widetilde{S}|^{2}}} .
\end{aligned}
$$

Observing that $\mu$ can be decomposed as

$$
\mu=\langle\mu, \mathbf{n}\rangle \mathbf{n}+\left\langle\mu, e_{n+1}\right\rangle e_{n+1}
$$

and $\left\langle\partial^{2} \widetilde{S} / \partial z_{i} \partial z_{j}, e_{n+1}\right\rangle=0$, we, then, have

$$
\begin{aligned}
A^{\Sigma}\left(\frac{\partial \widetilde{S}}{\partial z_{i}}, \frac{\partial \widetilde{S}}{\partial z_{j}}\right) & =-\frac{l \cos \angle(\mu, \mathbf{n})}{\sqrt{1+|\widetilde{S}|^{2}}}\left\langle\mathbf{n}, \frac{\partial^{2} \widetilde{S}}{\partial z_{i} \partial z_{j}}\right\rangle \\
& =\frac{l}{1+|\widetilde{S}|^{2}} A^{\widetilde{S}}\left(\frac{\partial \widetilde{S}}{\partial z_{i}}, \frac{\partial \widetilde{S}}{\partial z_{j}}\right),
\end{aligned}
$$

where we use the fact that $\cos \angle(\mu, \mathbf{n})=1 / \sqrt{1+|\widetilde{S}|^{2}}$.

\section{Evolution Equations and Boundary Derivatives}

In this section, we will derive evolution equations for some useful geometric quantities by straightforward calculation. Let $\left\{y_{1}, \ldots, y_{n}\right\}$ be the local normal coordinates of the evolving hyperserface and let $g_{i j}$ be the corresponding induced 
metric; let $\bar{\nabla}$ and $\bar{\Delta}$ be, respectively, the covariant derivative and Laplace operator with respect to the induced metric $g_{i j}$; and $|F|$ and $|A|$ denote the norm of the position vector and the second fundamental form to evolving hypersurfaces $M^{n}$ in $\mathbb{R}^{n+1}$, respectively.

Lemma 3. Under the mean curvature type flow (4), we have

(i) $(d / d t-u \bar{\Delta})|F|^{2}=0$,

(ii) $d v / d t=-\bar{\nabla}(n-H u)$,

(iii) $(d / d t-u \bar{\Delta}) u=n-2 H u+|A|^{2} u^{2}+H\langle\bar{\nabla} u, F\rangle$.

Proof. (i) We can calculate directly as in [14]

$$
\frac{d|F|^{2}}{d t}=2\left\langle\frac{d F}{d t}, F\right\rangle=2\langle(n-H u) v, F\rangle=2(n-H u) u .
$$

On the other hand,

$$
\begin{aligned}
\bar{\Delta}|F|^{2} & =g^{i j} \bar{\nabla}_{i} \bar{\nabla}_{j}\langle F, F\rangle=2 g^{i j} \bar{\nabla}_{i}\left\langle\frac{\partial F}{\partial y_{j}}, F\right\rangle \\
& =2 n-2 g^{i j}\left\langle h_{i j} \nu, F\right\rangle=2(n-H u) .
\end{aligned}
$$

This proves (i).

(ii) As $\langle\nu, v\rangle=1$, we have $\langle d \nu / d t, v\rangle=0$, and then,

$$
\begin{aligned}
\frac{d \nu}{d t} & =\left\langle\frac{d \nu}{d t}, \frac{\partial F}{\partial y_{i}}\right\rangle g^{i j} \frac{\partial F}{\partial y_{j}}=-\left\langle\nu, \frac{\partial}{\partial y_{i}} \frac{\partial F}{\partial t}\right\rangle g^{i j} \frac{\partial F}{\partial y_{j}} \\
& =-\frac{\partial}{\partial y_{i}}(n-H u) g^{i j} \frac{\partial F}{\partial y_{j}}=-\bar{\nabla}(n-H u) .
\end{aligned}
$$

(iii) Similarly, as in (i),

$$
\begin{aligned}
\frac{d u}{d t} & =\frac{d}{d t}\langle F, v\rangle=\left\langle\frac{d F}{d t}, v\right\rangle+\left\langle F, \frac{d v}{d t}\right\rangle \\
& =(n-H u)-\langle F, \bar{\nabla}(n-H u)\rangle \\
\bar{\Delta} u & =g^{i j} \bar{\nabla}_{i} \bar{\nabla}_{j}\langle F, v\rangle=g^{i j} \bar{\nabla}_{i}\left(\left\langle\frac{\partial F}{\partial y_{j}}, v\right\rangle+\left\langle F, h_{j k} \frac{\partial F}{\partial y_{k}}\right\rangle\right) \\
& =g^{i j} g_{i k} h_{j k}+\langle F, \bar{\nabla} H\rangle-h_{j k} h_{i l} g^{i j} g^{k l} u \\
& =H+\langle F, \bar{\nabla} H\rangle-|A|^{2} u .
\end{aligned}
$$

Then, (iii) follows by combining the above two formulae.

The following relationship between $A^{\Sigma}$ and $A$ was proved by Stahl in [3].

Lemma 4 (see [3]). For $p \in \partial M \times[0, T)$, one has

$$
A^{\Sigma}(\xi, \nu)=-A(\xi, \mu)
$$

where $\xi \in T_{p} M \cap T_{p} \Sigma$.
In order to apply the Hopf maximal principle to obtain the basic estimates, we also need the following boundary derivatives.

Lemma 5. For $(y, t) \in \partial M \times[0, T)$, one has
(i) $\left\langle\bar{\nabla}|F|^{2}, \mu\right\rangle=0$,
(ii) $\langle\bar{\nabla} u, \mu\rangle=u A^{\Sigma}(\nu, \nu)$.

Proof. (i) Denote by $\widetilde{\nabla}$ the Euclidean covariant derivative in $\mathbb{R}^{n+1}$. Obviously, $\widetilde{\nabla}|F|^{2} \in T_{p} \Sigma$; combining the boundary condition in (4), we have

$$
\left\langle\bar{\nabla}|F|^{2}, \mu\right\rangle=\left\langle\widetilde{\nabla}|F|^{2}-\left\langle\widetilde{\nabla}|F|^{2}, \nu\right\rangle \nu, \mu\right\rangle=\left\langle\widetilde{\nabla}|F|^{2}, \mu\right\rangle=0 .
$$

(ii) Calculating directly, we obtain

$$
\begin{gathered}
\bar{\nabla} u=\left\langle F, \frac{\partial v}{\partial y_{i}}\right\rangle g^{i j} \frac{\partial}{\partial y_{j}}=A\left(F^{\top}, \frac{\partial}{\partial y_{i}}\right) g^{i j} \frac{\partial}{\partial y_{j}}, \\
\langle\bar{\nabla} u, \mu\rangle=\left\langle A\left(F^{\top}, \frac{\partial}{\partial y_{i}}\right) g^{i j} \frac{\partial}{\partial y_{j}}, \mu\right\rangle=A\left(F^{\top}, \mu\right) .
\end{gathered}
$$

Observing $F^{\top}=F-u v$, combination of Lemma 4 and (i) in Proposition 2 yields

$$
\begin{aligned}
\langle\bar{\nabla} u, \mu\rangle & =-A^{\Sigma}(F-u v, \nu)=-A^{\Sigma}(F, \nu)+u A^{\Sigma}(\nu, \nu) \\
& =u A^{\Sigma}(\nu, \nu) .
\end{aligned}
$$

\section{Gradient Estimate}

Let $a_{i j}(y, t) \in L^{\infty}\left(M^{n} \times[0, T)\right)$ be a positive definite matrix such that

$$
\mathscr{L} f=\frac{d f}{d t}-a^{i j} \bar{\nabla}_{i} \bar{\nabla}_{j} f
$$

is a parabolic operator. We have the following maximal principle from Hopf Lemma [15] or Stahl's corresponding result in [4].

Theorem 6. Suppose $f: M^{n} \times[0, T) \rightarrow \mathbb{R}$ satisfies

$$
\begin{array}{r}
\frac{d f}{d t}-a^{i j} \bar{\nabla}_{i} \bar{\nabla}_{j} f(y, t) \leq 0 \\
\forall(y, t) \in M^{n} \times[0, T) \text { s.t. } \bar{\nabla} f(y)=0, \\
\langle\bar{\nabla} f, \mu\rangle \leq 0 \quad \forall(y, t) \in \partial M^{n} \times[0, T),
\end{array}
$$

and then $f(y, t) \leq \sup _{y \in M^{n}} f(y, 0)$ for all $(y, t) \in M^{n} \times[0, T)$.

Now we apply the above theorem to $|F|^{2}$. Combining (i) in Lemma 3 and (i) in Lemma 5, we immediately have the following estimates.

Corollary 7. Let $F(y, t)$ be a solution to system (4); then, one has

$$
\min _{y \in M_{0}^{n}}\left|F_{0}(y)\right| \leq|F(y, t)| \leq \max _{y \in M_{0}^{n}}\left|F_{0}(y)\right|
$$

for any $(y, t) \in M^{n} \times[0, T)$. 
This result means that the evolving hypersurface always stays between two spheres with radii $R_{1}=\max _{y \in M_{0}^{n}}\left|F_{0}(y)\right|$ and $R_{2}=\min _{y \in M_{0}^{n}}\left|F_{0}(y)\right|$.

In order to obtain the gradient estimate for system (12), we only need to estimate the lower bound for $u=\rho / \omega=$ $\rho / \sqrt{1+|\nabla r|^{2}}$. From (iii) in Lemma 3 ,

$$
\begin{aligned}
\left(\frac{d}{d t}-u \bar{\Delta}\right) u & =n-2 H u+|A|^{2} u^{2}+H\langle\bar{\nabla} u, F\rangle \\
& \geq(n-H u)-\frac{H u}{n}(n-H u)+H\langle\bar{\nabla} u, F\rangle \\
& =\frac{1}{n}(n-H u)^{2}+H\langle\bar{\nabla} u, F\rangle .
\end{aligned}
$$

On the other hand, noticing $\langle\mu, \nu\rangle=0$ by assumption, $v$ must be in the tangent space of the boundary cone; that is, $v \in T \Sigma$. Combining (ii) in Lemma 5, Proposition 2, and the convexity of the boundary cone, we have

$$
\langle\bar{\nabla} u, \mu\rangle=u A^{\Sigma}(\nu, \nu) \geq 0 .
$$

Equivalently,

$$
\begin{gathered}
\left(\frac{d}{d t}-u \bar{\Delta}\right)(-u) \leq-\frac{1}{n}(n-H u)^{2} \leq 0 \\
\forall(y, t) \in M^{n} \times[0, T) \quad \text { s.t. } \bar{\nabla} u(y, t)=0, \\
\langle\bar{\nabla}(-u), \mu\rangle=-u A^{\Sigma}(\nu, v) \leq 0 \quad \forall(y, t) \in \partial M^{n} \times[0, T) .
\end{gathered}
$$

By the maximal principle we have the following.

Corollary 8. For all $(y, t) \in M^{n} \times[0, T)$, the support function $u(y, t)$ satisfies

$$
u(y, t) \geq \min _{y \in M^{n}} u(y, 0) .
$$

Combining Corollaries 7 and 8, we obtain long time existence for the system (12) by the standard argument for divergence PDE (cf. [13]), and then, the first part of Theorem 1 follows by the equivalence of (4) and (12).

Let $V$ be the domain enclosed by the interior of the cone and the evolving hypersurface. The volume element for $V$ is denoted by $d V$ and $d V_{\partial}$ for its boundary. We will also denote the area elements for the evolving hypersurface and its boundary by $d v$ and $d v_{\partial}$, respectively. Flow (4) has the following interesting property.

Proposition 9. Along flow (4), the volume of $V$, denoted by $|V|$, is a constant.

Proof. For the function $\Phi(y, t)=(1 / 2)|F(y, t)|^{2}$ defined on the evolving hypersurface $M_{t}$, we have

$$
\bar{\Delta} \Phi=n-H u
$$

from the proof of Lemma 3(i). Integrating the above equation on $M_{t}$ and taking into consideration Lemma 5(i) yield

$$
\begin{aligned}
\int_{M_{t}}(n-H u) d v & =\int_{M_{t}} \bar{\Delta} \Phi d v=\int_{\partial M_{t}}\langle\bar{\nabla} \Phi, \mu\rangle d v_{\partial} \\
& =\frac{1}{2} \int_{\partial M_{t}}\left\langle\bar{\nabla}|F|^{2}, \mu\right\rangle d v_{\partial}=0 .
\end{aligned}
$$

Denote by $\widetilde{\operatorname{div}} X$ the divergence of the position vector $X$ for $V$ in $\mathbb{R}^{n+1}$; we have

$$
\int_{V} \widetilde{\operatorname{div}} X d V=(n+1)|V| .
$$

Taking derivative with respect to time $t$ and combining divergence theorem yield

$$
\begin{aligned}
(n+1) \frac{d}{d t}|V| & =\int_{V} \widetilde{\operatorname{div}}\left(\frac{d X}{d t}\right) d V=\int_{\partial V}\left\langle\frac{d X}{d t}, \eta\right\rangle d V_{\partial} \\
& =\int_{\Sigma}\left\langle\frac{d X}{d t}, \mu\right\rangle d v_{\Sigma}+\int_{M_{t}}\left\langle\frac{d X}{d t}, v\right\rangle d v \\
& =\int_{M_{t}}(n-H u) d v=0,
\end{aligned}
$$

where $\eta$ is the outward unit normal vector to the $(n+$ 1)-dimensional region $V$, and $d v_{\Sigma}$ is the area element on boundary cone $\Sigma$. Hence, $|V|$ is a constant and the result follows.

\section{Convergence}

In this section, we use an idea of Guan and Li in [1] to obtain our estimate and prove the exponential convergence. For that purpose, the system (9) or (12) is convenient for us. Now, we choose a local coordinate $\left\{x_{1}, \ldots, x_{n}\right\}$ on $\Omega \subset \mathbb{S}^{n}$. $\nabla$ and $\Delta$ are again the covariant derivative and Laplace operator with respect to the standard metric $e_{i j}$ on $\mathbb{S}^{n}$. Let $\mathfrak{Q}$ be a parabolic operator defined by

$$
\mathfrak{L} f=\frac{d f}{d t}-\frac{1}{\rho \omega}\left(e^{i j}-\frac{\nabla^{i} r \nabla^{j} r}{\omega^{2}}\right) f_{i j}
$$

on $\Omega \times[0, T)$ for a smooth function $f: \Omega \times[0, T) \rightarrow \mathbb{R}$. From [1], we have the following evolution equation for $|\nabla r|^{2} / 2$ at the critical point

$$
\begin{aligned}
\mathfrak{L}\left(\frac{|\nabla r|^{2}}{2}\right)= & -\frac{n}{\rho \omega}|\nabla r|^{4}-\frac{n-1}{\rho \omega}|\nabla r|^{2} \\
& -\frac{1}{\rho \omega} \Delta r|\nabla r|^{2}-\frac{1}{\rho \omega}\left|\nabla^{2} r\right|^{2} .
\end{aligned}
$$

Using Cauchy-Schwartz inequality and similar rearrangement as in [10], we have

$$
\begin{aligned}
\mathfrak{Q}|\nabla r|^{2} \leq & -\frac{3 n}{2 \rho \omega}|\nabla r|^{4}-\frac{2(n-1)}{\rho \omega}|\nabla r|^{2} \\
& -\frac{2}{n \rho \omega}\left(\Delta r+\frac{n}{2}|\nabla r|^{2}\right)^{2} .
\end{aligned}
$$


Therefore,

$$
\mathfrak{L}|\nabla r|^{2} \leq-\frac{2(n-1)}{\rho \omega}|\nabla r|^{2} \leq 0 .
$$

Recall that $\rho=\omega u$ and $\omega=\sqrt{1+|\nabla r|^{2}}$; we have, by Lemma 5 and the Neumann boundary assumption, for any points $(x, t) \in \partial \Omega \times[0, T)$,

$$
\begin{aligned}
\left\langle\nabla\left(|\nabla r|^{2}\right), \mu\right\rangle= & \left\langle\nabla\left(\omega^{2}-1\right), \mu\right\rangle \\
= & \frac{2 \rho}{u^{2}}\langle\nabla \rho, \mu\rangle \\
& -\frac{2 \rho^{2}}{u^{3}}\langle\nabla u, \mu\rangle=-\frac{2 \rho^{2}}{u^{2}} A^{\Sigma}(\nu, \nu) .
\end{aligned}
$$

With the assumption of convexity on the boundary cone, we obtain, by Theorem 6 ,

$$
|\nabla r|^{2}(x, t) \leq \max _{x \in \Omega}|\nabla r|^{2}(x, 0) .
$$

By the equivalence of (4) and (9), we have

$$
\min _{x \in \Omega} \rho(x, 0) \leq \rho(x, t) \leq \max _{x \in \Omega} \rho(x, 0)
$$

from Corollary 7.

From the above estimate, there exists a uniform positive constant $\alpha \leq 2(n-1) / \rho \omega$ depending only on the upper bound of $\omega$ and $\rho$ such that (41) reads as

$$
\mathfrak{Q}|\nabla r|^{2} \leq-\alpha|\nabla r|^{2} .
$$

Denote by $g=e^{\alpha t}|\nabla r|^{2}$

$$
\begin{aligned}
\mathfrak{L} g & =\mathfrak{L}\left(e^{\alpha t}|\nabla r|^{2}\right)=\alpha e^{\alpha t}|\nabla r|^{2}+e^{\alpha t} \mathfrak{Q}|\nabla r|^{2} \leq 0, \\
\langle\nabla g, \mu\rangle & =e^{\alpha t}\left\langle\nabla \omega^{2}, \mu\right\rangle=-2 \frac{\rho^{2}}{u^{2}} e^{\alpha t} A^{\Sigma}(\nu, \nu) \leq 0 .
\end{aligned}
$$

Then, by Theorem 6 again, $g=e^{\alpha t}|\nabla r|^{2}$ has uniform upper bound $C_{1}=\max _{x \in \Omega} g(x, 0)$; that is

$$
|\nabla r|^{2} \leq C_{1} e^{-\alpha t}, \quad t \in[0, \infty) .
$$

Or, equivalently, for a different constant $C_{2}=\rho_{\max _{x \in \Omega}}^{2}(x, 0) C_{1}$,

$$
|\nabla \rho|^{2} \leq C_{2} e^{-\alpha t}, \quad t \in[0, \infty) .
$$

This means that $M_{t}$ converges exponentially fast to some part of a sphere.

Assume the radial function $\rho\left(x, t_{0}\right)$ attains its minimum at a point $p \in \Omega$ for some $t_{0} \in[0, \infty)$; that is, $\rho\left(p, t_{0}\right)=$ $\rho_{\min _{x \in \Omega}}\left(x, t_{0}\right)$. Let $\sigma:[0, s) \rightarrow \Omega$ be a geodesic on the unit sphere $\mathbb{S}^{n}$ starting from $p$ to any point $x \in \Omega$ with $\sigma(s)=$ $x$. Integrating both sides of the last inequality on $[0, s]$ and taking into account the boundedness of $s$, we have, for some constant $C_{3}=C_{3}\left(\Omega, C_{2}, \alpha\right)$ and $\beta=\alpha / 2$,

$$
\int_{0}^{s} \frac{d \rho}{d s} d s \leq \int_{0}^{s}|\nabla \rho| d s \leq C_{3} e^{-\beta t_{0}}
$$

and then,

$$
\rho\left(x, t_{0}\right)-\rho\left(p, t_{0}\right)=\rho\left(x, t_{0}\right)-\rho_{\min _{x \in \Omega}}\left(x, t_{0}\right) \leq C_{3} e^{-\beta t_{0}} .
$$

So we have

$$
\rho_{\max _{x \in \Omega}}(x, t)-\rho_{\min _{x \in \Omega}}(x, t) \leq C_{3} e^{-\beta t}, \quad t \in[0, \infty) .
$$

On the other hand,

$$
\begin{aligned}
(n+1)|V| & =\int_{V} \widetilde{\operatorname{div}} X d V=\int_{\partial V}\langle X, \eta\rangle d V_{\partial} \\
& =\int_{\Sigma}\langle X, \mu\rangle d v_{\Sigma}+\int_{M_{t}}\langle X, \nu\rangle d v=\int_{M_{t}} u d v .
\end{aligned}
$$

Because

$$
\begin{gathered}
\rho_{\max _{x \in \Omega}}(x, t)-C_{3} e^{-\beta t} \leq \rho(x, t) \leq \rho_{\min _{x \in \Omega}}(x, t)+C_{3} e^{-\beta t}, \\
1 \leq \omega=\sqrt{1+|\nabla r|^{2}} \leq \sqrt{1+C_{1} e^{-\alpha t}}
\end{gathered}
$$

the support function $u=\rho / \omega$ satisfies

$$
\frac{\rho_{\max _{x \in \Omega}}(x, t)-C_{3} e^{-\beta t}}{\sqrt{1+C_{1} e^{-\alpha t}}} \leq u \leq \rho_{\min _{x \in \Omega}}(x, t)+C_{3} e^{-\beta t} .
$$

Denote by $\left|M_{t}\right|$ the area of $M_{t}$; combining (52), we have

$$
\begin{aligned}
\left|M_{t}\right| \frac{\rho_{\max _{x \in \Omega}}(x, t)-C_{3} e^{-\beta t}}{\sqrt{1+C_{1} e^{-\alpha t}}} & \leq(n+1)|V| \\
& \leq\left|M_{t}\right|\left(\rho_{\min _{x \in \Omega}}(x, t)+C_{3} e^{-\beta t}\right),
\end{aligned}
$$

or, equivalently,

$$
\begin{aligned}
& \frac{(n+1)|V|}{\rho_{\min _{x \in \Omega}}(x, t)+C_{3} e^{-\beta t}} \\
& \quad \leq\left|M_{t}\right| \leq(n+1)|V| \frac{\sqrt{1+C_{1} e^{-\alpha t}}}{\rho_{\max _{x \in \Omega}}(x, t)-C_{3} e^{-\beta t}} .
\end{aligned}
$$

Let $\lim _{t \rightarrow \infty} \rho(x, t)=\rho(x)$, and from the above inequality, we obtain $\rho(x)=R$, a constant, and therefore,

$$
\left|M_{t}\right| \longrightarrow \frac{(n+1)|V|}{R}, \quad t \longrightarrow \infty .
$$

That is to say, $M_{t}$ converges exponentially to the intersection of the interior of the cone $\Sigma$ and a sphere centered at the vertex of the cone with radius

$$
R=\frac{(n+1)|V|}{\left|M_{\infty}\right|}
$$

where $\left|M_{\infty}\right|$ denotes the area of the limit sphere $M_{\infty}$. This finishes the proof of Theorem 1. 


\section{Conflict of Interests}

The authors declare that there is no conflict of interests regarding the publication of this paper.

\section{Acknowledgments}

The research is partially supported by NSFC (no. 11171096), RFDP (no. 20104208110002), and Funds for Disciplines Leaders of Wuhan (no. Z201051730002).

\section{References}

[1] P. Guan and J. Li, "A mean curvature type flow in space forms," International Mathematics Research Notices, 2014.

[2] G. Huisken, "Nonparametric mean curvature evolution with boundary conditions," Journal of Differential Equations, vol. 77, no. 2, pp. 369-378, 1989.

[3] A. Stahl, "Convergence of solutions to the mean curvature flow with a Neumann boundary condition," Calculus of Variations and Partial Differential Equations, vol. 4, no. 5, pp. 421-441, 1996.

[4] A. Stahl, "Regularity estimates for solutions to the mean curvature flow with a Neumann boundary condition," Calculus of Variations and Partial Differential Equations, vol. 4, no. 4, pp. 385-407, 1996.

[5] J. A. Buckland, "Mean curvature flow with free boundary on smooth hypersurfaces," Journal für die Reine und Angewandte Mathematik, vol. 586, pp. 71-90, 2005.

[6] B. Lambert, "The perpendicular Neumann problem for mean curvature flow with a timelike cone boundary condition," Transactions of the American Mathematical Society, vol. 366, no. 7, pp. 3373-3388, 2014.

[7] B. Lambert, "The constant angle problem for mean curvature flow inside rotational tori," http://arxiv.org/abs/1207.4422.

[8] D. Hartley, "Motion by volume preserving mean curvature flow near cylinders," Communications in Analysis and Geometry, vol. 21, no. 5, pp. 873-889, 2013.

[9] S. J. Altschuler and L. F. Wu, "Translating surfaces of the nonparametric mean curvature flow with prescribed contact angle," Calculus of Variations and Partial Differential Equations, vol. 2, no. 1, pp. 101-111, 1994.

[10] B. Guan, "Mean curvature motion of non-parametric hypersurfaces with contact angle condition," in Elliptic and Parabolic Methods in Geometry, pp. 47-56, A. K. Peters, Wellesley, Mass, USA, 1996.

[11] G. Li and I. Salavessa, "Forced convex mean curvature flow in Euclidean spaces," Manuscripta Mathematica, vol. 126, no. 3, pp. 333-351, 2008.

[12] X. Zhu, Lectures on Mean Curvature Flows, vol. 32 of Studies on Advanced Mathematics, American Mathematical Society, International Press, 2002.

[13] G. Lieberman, Second Order Parabolic Differential Equations, World Scientific Publishing, 1996.

[14] K. Ecker and G. Huisken, "Mean curvature evolution of entire graphs," Annals of Mathematics, vol. 130, no. 3, pp. 453-471, 1989.

[15] M. Protter and H. Weinberger, Maximum Principle in Differential Equation, Springer, 1984. 


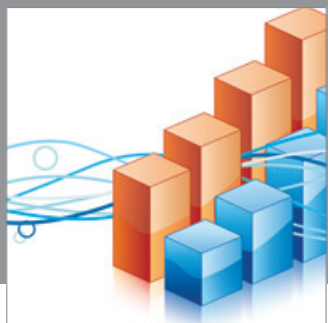

Advances in

Operations Research

mansans

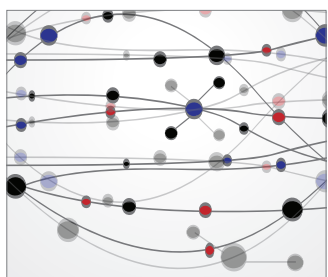

The Scientific World Journal
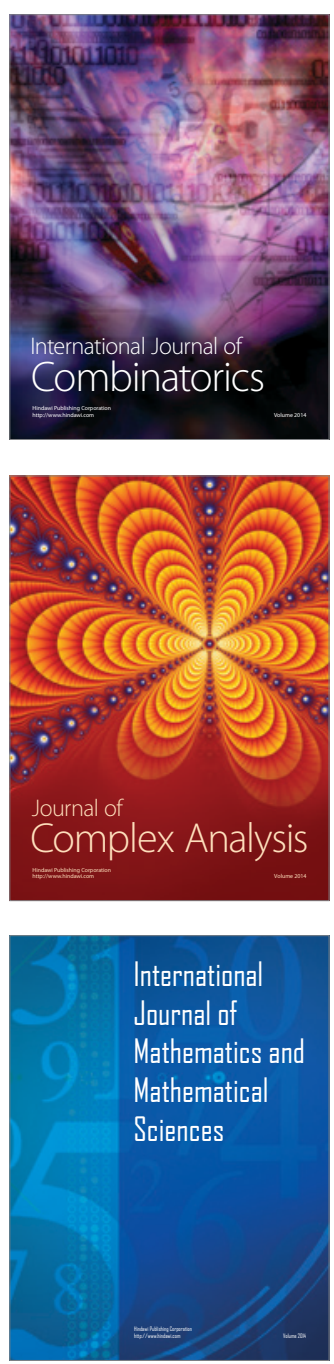
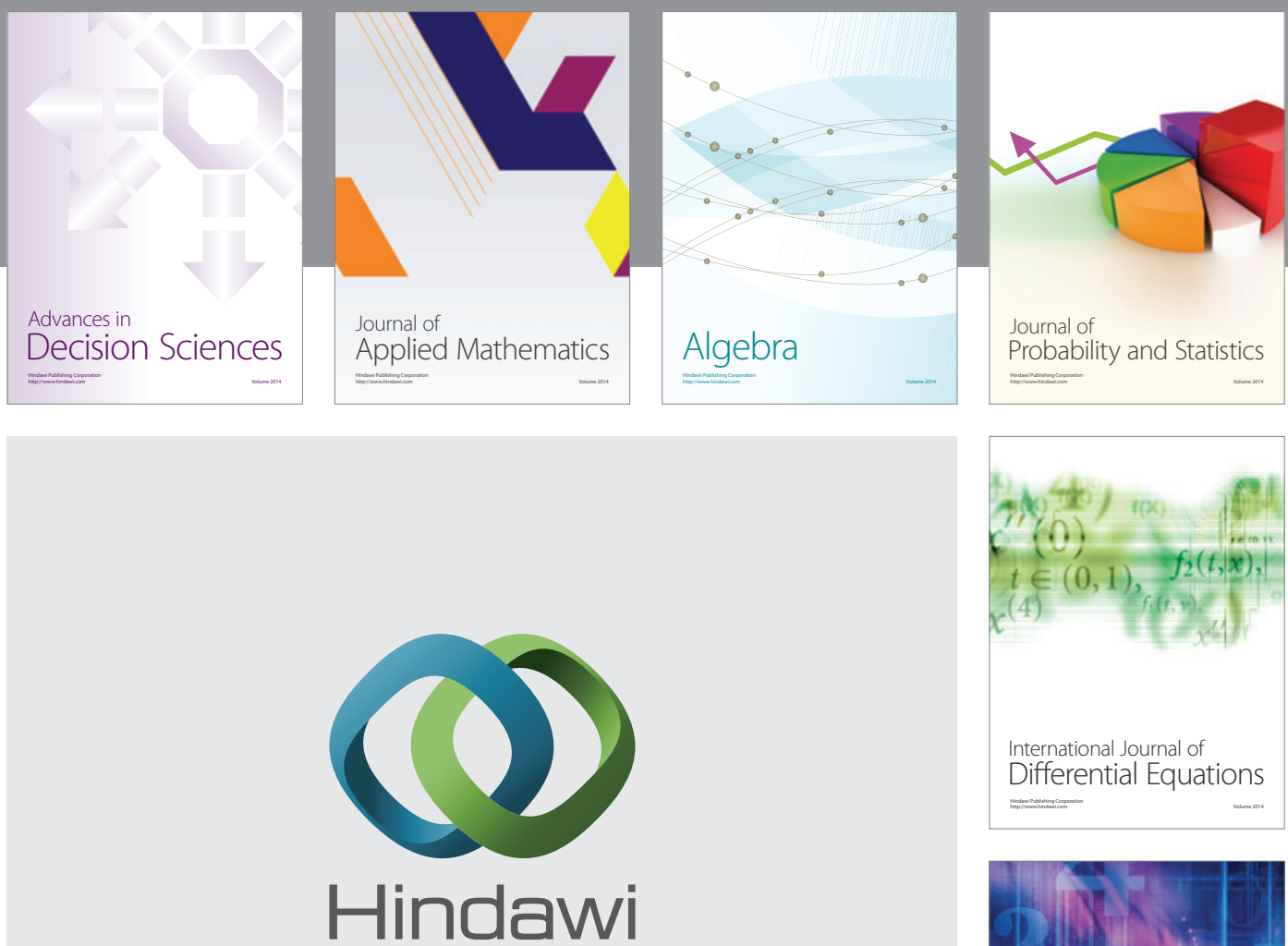

Submit your manuscripts at http://www.hindawi.com
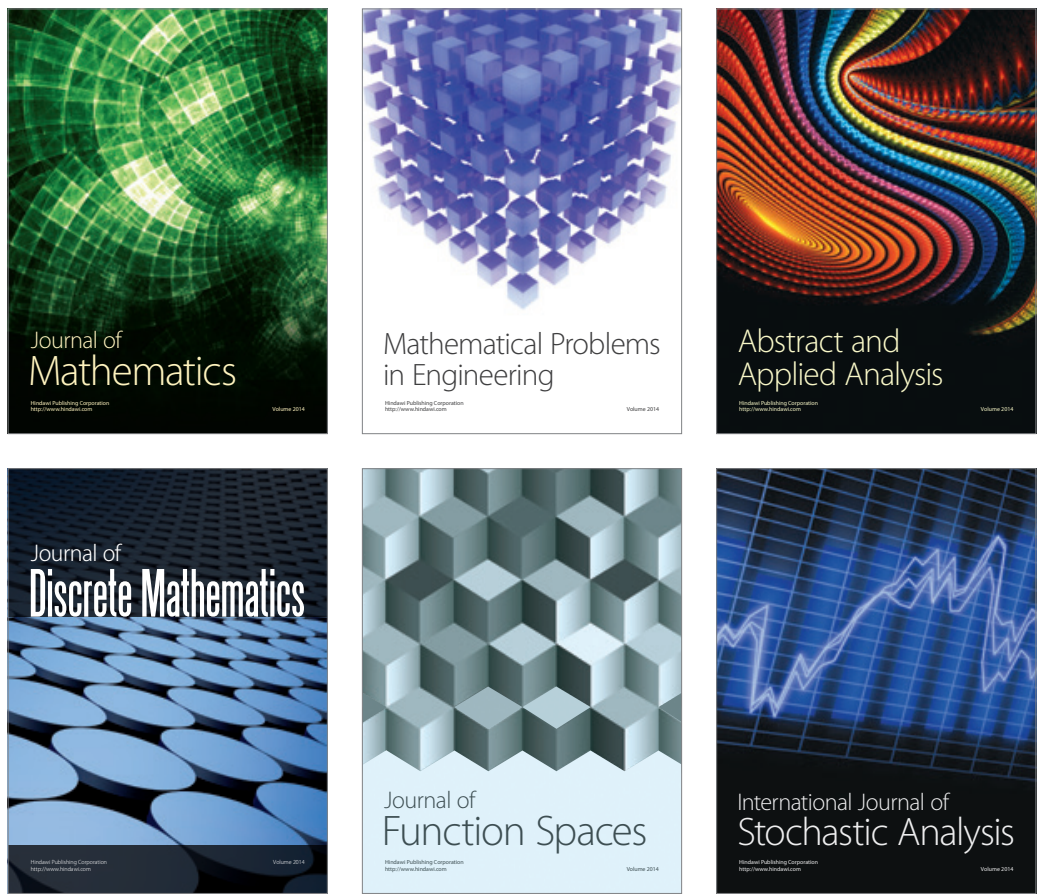

Journal of

Function Spaces

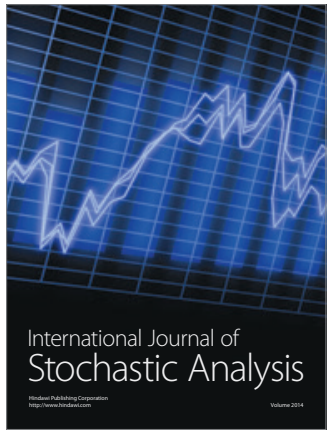

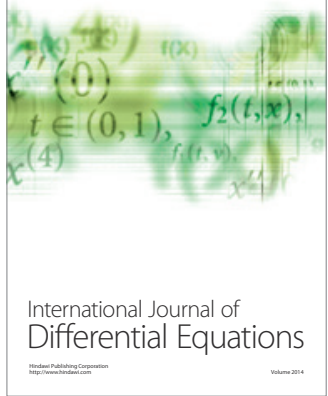
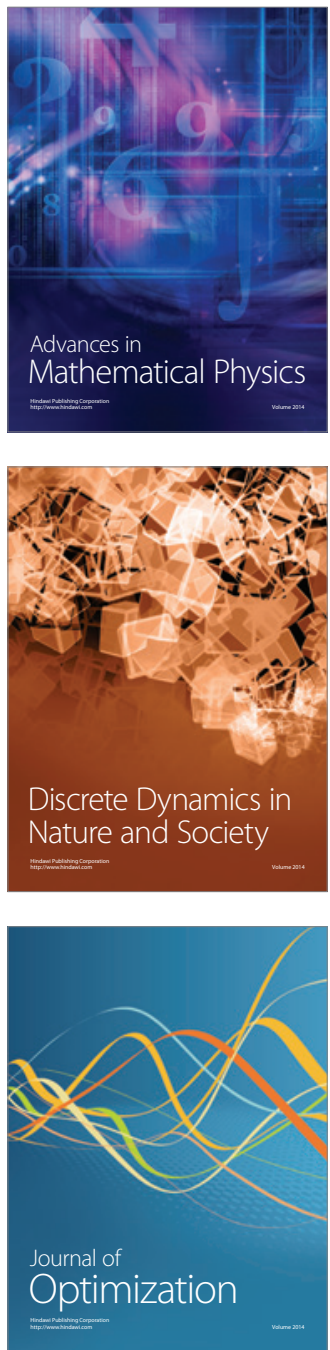